\title{
Erratum to: Selection of Large-Scale 3D Point Cloud Data Using Gesture Recognition
}

Robin Burgess $^{(\bowtie)}$, António J. Falcão, Tiago Fernandes, Rita A. Ribeiro, Miguel Gomes, Alberto Krone-Martins, and André Moitinho de Almeida

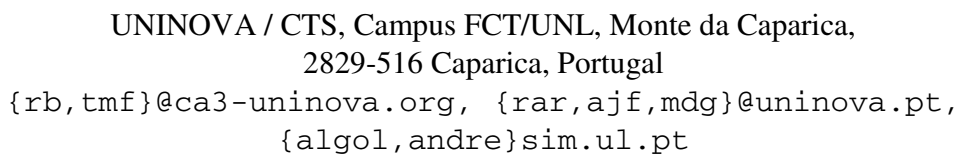

UNINOVA / CTS, Campus FCT/UNL, Monte da Caparica,

2829-516 Caparica, Portugal

$\{r b, t m f\} @$ ca3-uninova.org, \{rar, ajf, mdg\} @uninova.pt,

\{algol, andre\}sim.ul.pt

\section{Erratum to:}

\section{Chapter 20 in: L.M. Camarinha-Matos et al. (Eds.) Technological Innovation for Cloud-Based Engineering Systems, DOI: 10.1007/978-3-319-16766-4_20}

In the original publication the legends of Figs. 1 and 2 did not include the statement that they were used with permission from IEEE. The correct statement is:

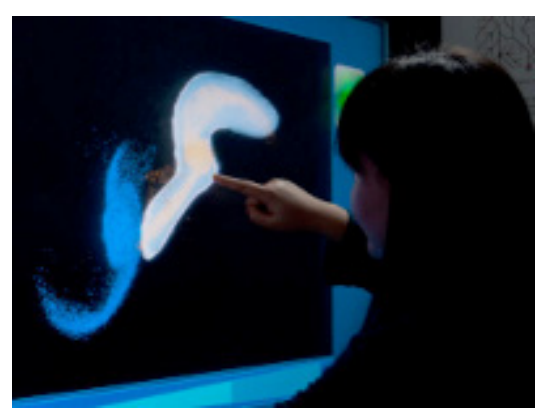

Fig. 1. 3D selection using a touch-based interface [5]. (C) 2015 IEEE. Reprinted, with permission, from Yu, L., Efstathiou, K., Isenberg, P., Isenberg, T.: Efficient Structure-Aware Selection Techniques for 3D Point Cloud Visualizations with 2DOF Input. IEEE Transactions on Visualization and Computer Graphics 18(12), 2245-2254 (2012) 


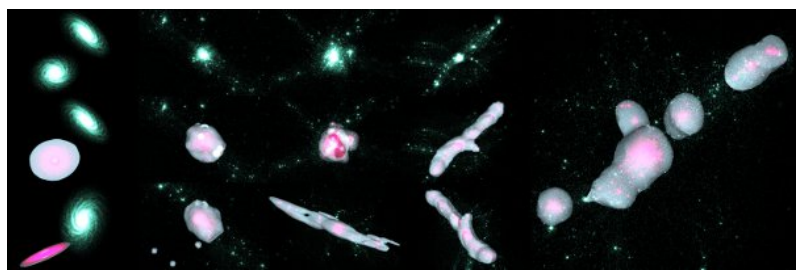

Fig. 2. 3D selection of galaxies using TeddySelection and CloudLasso techniques [5]. (C) 2015 IEEE. Reprinted, with permission, from Yu, L., Efstathiou, K., Isenberg, P., Isenberg, T.: Efficient Structure-Aware Selection Techniques for 3D Point Cloud Visualizations with 2DOF Input. IEEE Transactions on Visualization and Computer Graphics 18(12), 2245-2254 (2012) 